\title{
El Proyecto Monitor como estrategia de nivelación en la universidad The Instructor Project as a leveling strategy at the university
}

\author{
Domingo A. Martín Sánchez ${ }^{1}$, Leticia Presa Madrigal², Ana García Laso ${ }^{3}$, Jorge L. Costafreda Mustelier ${ }^{4}$, \\ David Bolonio Martín ${ }^{5}$ \\ domingoalfonso.martin@upm.es, leticia.presa.madrigal@alumnos.upm.es, ana.garcia.laso@gmail.com, \\ jorgeluis.costafreda@gmail.com,david.bolonio@upm.es \\ ${ }^{1}$ Adjunto Asuntos Económicos e Infraestructuras \\ E.T.S.I de Minas y Energía de la UPM \\ Madrid, España \\ ${ }^{2}$ Unidad de Emprendimiento Social Ética y Valores \\ E.T.S.I de Minas y Energía de la UPM \\ Madrid, España
}

\begin{abstract}
Resumen- Uno de los cambios que ha supuesto la convergencia de las titulaciones hacia el Espacio Europeo de Educación Superior (EEES) ha sido la modificación de los horarios con el desplazamiento de la convocatoria de septiembre a julio. Si sumamos a este hecho el comienzo prematuro de los cursos académicos a principios del mes de septiembre estamos dificultando la implantación de las medidas de nivelación que las universidades diseñaban para nivelar a los alumnos de primer curso con distintas procedencias. Esta nivelación académica se realizaba en la Universidad Politécnica de Madrid (UPM) a través del curso "cero". Esta situación hace que algunos de nuestros alumnos tengan dificultades para superar las asignaturas de primer curso por ser de un nivel de exigencia alto. Una estrategia que parte de los propios alumnos a través de una actividad formativa de emprendimiento social que se desarrolla en nuestro centro es el proyecto monitor. En esta comunicación vamos a medir el diferente grado de impacto que tiene sobre los alumnos esta actuación basada en la cooperación entre iguales coordinadas por los diferentes profesores de esas asignaturas. Se analizarán la tasa de eficiencia, éxito y la de absentismo entre otras variables y podremos cuantificar los beneficios que se producen con este tipo de actuaciones
\end{abstract}

Palabras clave: Proyecto Monitor, nivelación académica, tasa de éxito, eficiencia y absentismo.

Abstract- One of the changes that have led to the convergence of degrees towards the EHEA has been the modification of the schedules, displacing the examinations from September to July. This added to the fact the premature beginning of the academic courses begin prematurely at the beginning of September, the implementation of the leveling measures that the universities designed to level the first-year students from different origins is made increasingly difficult. This academic leveling was done at the Universidad Politécnica de Madrid (UPM) through the "zero" course. Because of this situation, some students have difficulties to overcome the firstyear courses, especially the ones that demand high level of effort. In order to solve this problem, the instructor project is a training activity, introduced by the UPM, which starts from the students themselves and encourages the social entrepreneurship. In this work, the different level of impact that students have on this strategy is measured, based on their cooperation and the coordination between professors of the involved subjects. Variables such as the rate of efficiency, success and absenteeism are analyzed to quantify the benefits that occur when these actions are applied.

Keywords: Instructor project, academic level, success rate, efficiency and absenteeism.

\section{INTRODUCCIÓN}

La universidad española y concretamente los estudios de ingeniería se encuentran decalados, en cuanto a nivel de exigencia en los primeros cursos, con los conocimientos que los alumnos de nuevo ingreso traen desde sus distintas procedencias. Por otra parte los órganos de gobierno de las universidades van introduciendo cambios adaptativos en los planes de estudios universitarios con el fin de adaptarse al nuevo marco que supone la convergencia de las titulaciones hacia el Espacio Europeo de Educación Superior (EEES). En estos años de adaptación hemos podido observar la necesidad de incorporar un sistema de nivelación del grado de conocimiento previo de los alumnos. De esta forma se intenta evitar las dificultades que encuentran, nuestros alumnos, para superar las asignaturas de la titulación dado su alto grado de exigencia académica. Esta situación se manifiesta en determinadas asignaturas que se fundamentan de forma solida en unos conocimientos previos que el alumno debe obtener. En nuestro caso se pueden detectar estas asignaturas por suponer gran cumulo de alumnos suspensos con pocas expectativas de éxito. Estas asignaturas suelen estar relacionadas con los dos primeros años de grado de nuestros alumnos.

La implantación del EEES supone un reto y al mismo tiempo una oportunidad para proceder a implantar un conjunto de mejoras, innovaciones docentes y nuevas metodologías fruto de la reflexión (García, Ana María Delgado 2005)

Desde un punto de vista cognitivista, para el aprendizaje es necesario tener en cuenta no solo la enseñanza, sino las circunstancias del objeto de la enseñanza, del alumno, y entre ellas, principalmente, los conocimientos de los que el alumno parte cuando comienza una etapa de aprendizaje concreto (constructivismo). Este aspecto, trasladado a la programación de una materia con créditos europeos, implica que será necesaria una exigencia absoluta de dominio de los conocimientos que han debido de adquirirse en etapas anteriores, para que pueda alcanzarse el nivel adecuado de la materia en el tiempo programado. (Reyes Ruiz-Gallardo, José 2008;) 
Es por ello que desde la Unidad de Emprendimiento Social Ética y Valores en la Ingeniería (EUSEVI) de la Escuela de Minas y Energía (ETSIME) de la UPM se propuso la implantación de este proyecto que intenta suplir las carencias de los alumnos de nuevo ingreso que anteriormente se satisfacían mediante el curso "cero", cuya implantación queda imposibilitada por el temprano comienzo de los cursos académicos con el nuevo plan de estudio, lo cual imposibilita al alumno el acceso a las herramientas necesarias para superar el salto cualitativo de nivel entre el bachillerato y la universidad. Eduardo Martín Cabrera (1999) señala que “el estudiante universitario ha sido concebido tradicionalmente como el único responsable de su aprendizaje, y más concretamente, su voluntad y dedicación, con lo cual el éxito o el fracaso se debía a una decisión personal” limitando así “el desarrollo de sistemas de ayuda al estudiante”.

Esta iniciativa pretende incorporar la participación de alumnos que previamente han superado estas dificultados con un alto grado de satisfacción junto con los profesores de las asignaturas para así poder acercar las inquietudes de los alumnos y poder detectar las deficiencias en los conocimientos reforzando las áreas en las que se aprecian mayores dificultades

\section{CONTEXTO}

Tras apreciar la necesidad latente por parte de los profesores de un instrumento de nivelación y observando que los conocimientos de partida de los alumnos de nuevo ingreso eran insuficientes para el cumplimiento de las competencias especificas y los resultados de aprendizaje requeridos en algunas asignaturas, desde las actividades realizadas en la UESEVI dentro de la actividad formativa Emprendimiento e Innovación social se diseña el Proyecto Monitor como herramienta de convergencia entre ambos colectivos, alumnos y profesores.

Este nuevo proyecto se presenta como una actividad que complementa al Proyecto Mentor, el cual cubre las necesidades logísticas de los alumnos de nuevo ingreso, sin embargo este formato "tiene un carácter preventivo, dado que este tipo de actuaciones va encaminado, en el mejor de los casos, a evitar el fracaso escolar, la baja autoestima, la dispersión, o a aminorar sus efectos. Asimismo, se pretende que tenga carácter propedéutico, trabajando diversas técnicas que permitan optimizar el estudio y el aprendizaje del alumno de primer curso" tal como describen Sánchez García, M., Manzano Soto, N., Rísquez, A., \& Suárez Ortega, M. (2011).

El proyecto se engloba en un marco del afán de avanzar en el proceso de mejora continua en los procesos del Sistema de Garantía Interno de calidad (SGIC). Con esta iniciativa se ofrece una solución documentada a una casuística ya detectados en el alumnado, para poder garantizar un seguimiento óptimo de los procesos educativos que tienen lugar en la universidad.

\section{DESCRIPCIÓN}

El Proyecto Monitor comienza a principios del curso 2014/2015 con su implantación en cuatro asignaturas de primer año de Grado de Ingeniería de la Energía y Grado de ingeniería en Tecnología Minera. Como se puede ver en la tabla 1 se muestran las asignaturas en las que se ha implantado el proyecto en estos tres años.
Tabla 1

Asignaturas y años en las que se ha implantado el Proyecto Monitor. Fuente elaboración propia.

\begin{tabular}{ccc}
\hline 2014/15 & $2015 / 16$ & 2016/17 \\
\hline Expresión & Expresión & Expresión \\
Gráfica & Gráfica & Gráfica \\
Química I & Química I & Geología \\
Química II & Química II & Química II \\
Cálculo I & Estadística & Estadística \\
\hline
\end{tabular}

Se implantan dos formatos diferenciados, en el primer caso el refuerzo de conocimientos se realiza en el aula con la presencia del profesor de la asignatura, que obtiene un soporte para poder atender y resolver dudas de los alumnos consiguiendo un avance más rápido que permite personalizar el aprendizaje de cada alumno a sus necesidades individuales, este formato se aplica en asignaturas como Expresión Gráfica y Geología. El segundo formato consiste en impartir el refuerzo de las asignaturas fuera del horario lectivo, siempre contando con el apoyo de los profesores, esto permite a los alumnos resolver las dudas que les surgen durante el estudio, afianzar los conocimientos adquiridos y reforzar el estudio en aquellos conocimientos previos que deberían haberse adquirido en Bachillerato. Este formato se ha implantado en Química I, Química II, Cálculo I y Estadística.

El proceso de selección de los alumnos monitores es llevado a cabo, en ambos casos, por los profesores de las asignaturas. Los alumnos que hayan cursado la asignatura con un rendimiento elevado son preseleccionados y citados a una reunión con el profesorado y los coordinadores del proyecto, tras la cual se selecciona a los alumnos con mayor disposición.

La finalidad del proyecto es evitar tanto las dificultades encontradas por los alumnos por el enorme cambio que supone el paso del instituto a la universidad, como en niveles posteriores evitar el fenómeno conocido como "asignaturas cuello de botella”, en las cuales los alumnos tienen un bajo rendimiento académico por la falta de conocimientos requeridos para cursar esas asignaturas. Por ello el proyecto pretende abarcar en un futuro próximo asignaturas de cursos posteriores que se encuentran en esta situación, de esta forma durante el curso 2016/2017 se ha incorporado la asignatura de segundo curso de Geología, tras comprobar los resultados obtenidos en esta asignatura se decidirá si es conveniente ampliar el proyecto a cursos posteriores.

\section{Resultados}

Para la evaluación de los resultados obtenidos a lo largo de estos años se han utilizado diferentes indicadores:

- Tasa de rendimiento: Relación porcentual entre el $n^{\circ}$ de alumnos aprobados y el $\mathrm{n}^{\circ}$ de alumnos matriculados.

- Tasa de éxito: Relación porcentual entre el $n^{\circ}$ de alumnos aprobados y el $\mathrm{n}^{\circ}$ de alumnos presentados.

- Tasa de absentismo: Relación porcentual entre el $n^{\circ}$ de alumnos NO presentados y el $\mathrm{n}^{\circ}$ de alumnos

- $\quad$ Notas de acceso de los alumnos matriculados. 
En la tabla 2 se muestra la evolución de los resultados de la tasa de rendimiento, en porcentaje, en las distintas asignaturas que se cursan en el primer año de grado:

\section{Tabla 2}

Evolución temporal de la tasa de rendimiento en las distintas asignaturas que se cursan en el primer semestre de primer curso del Grado en Ingeniería de la Energía.. Fuente elaboración propia a partir de datos de los informes de la comisión de coordinación académica de curso de los años 2013 a 2017.

\begin{tabular}{|c|c|c|c|c|c|}
\hline \multicolumn{6}{|c|}{$\begin{array}{l}\text { RELACIÓN PORCENTUAL ENTRE N }{ }^{\circ} \text { DE ALUMNOS } \\
\text { APROBADOS Y N }{ }^{\circ} \text { DE ALUMNOS MATRICULADOS } \\
\text { GIE }\end{array}$} \\
\hline \multirow{2}{*}{\multicolumn{6}{|c|}{$\begin{array}{ccccc}2012 & 2013 / & 2014 / & 2015 / & 2016 / \\
2013 & 2014 & 2015 & 2016 & 2017\end{array}$}} \\
\hline & & & & & \\
\hline \multicolumn{6}{|c|}{$\begin{array}{llllll}\text { ALGEBRA } & 78.17 & 76.09 & 83.09 & 84.12 & 84.88\end{array}$} \\
\hline \multicolumn{6}{|l|}{ CALCULO I } \\
\hline \multicolumn{6}{|l|}{$\begin{array}{c}\text { EXPRESION } \\
\text { GRAFICA }\end{array}$} \\
\hline FISICA I & 73.03 & 85.71 & 84.83 & 67.98 & 59.57 \\
\hline QUIMICA I & 74.85 & 72.33 & 81.82 & 64.29 & 66.83 \\
\hline
\end{tabular}

Se puede observar cómo a partir del año 2014/2015 se produce una bajada de la tasa de rendimiento en asignaturas como Física y Calculo I mientras que en expresión Gráfica se produce un aumento de esta tasa.

Si estudiamos más a fondo la asignatura de Expresión Gráfica se observa cómo este ascenso en la tasa de rendimiento es evidente, esto se muestra en la Figura 1.

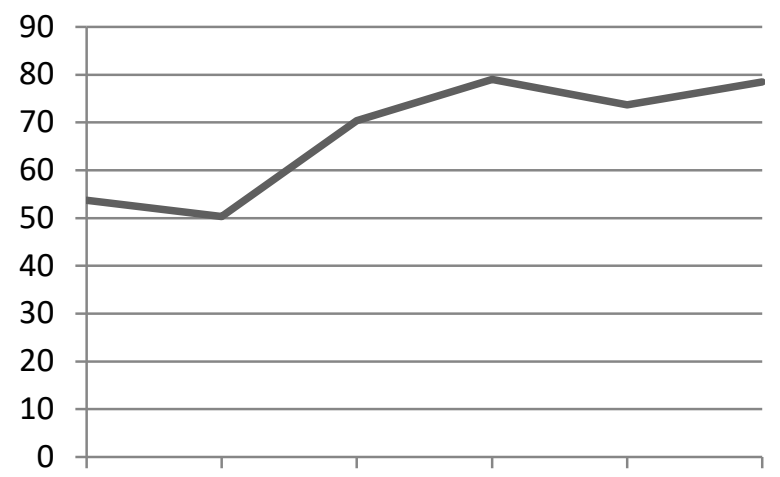

2011/12 2012/13 2013/14 2014/15 2015/16 2016/17

Figura 1. Evolución tasa de rendimiento de Expresión Gráfica en tanto por ciento. Fuente elaboración propia a partir de datos de los informes de la comisión de coordinación académica de curso de los años 2013 a 2017.

En la Figura 2 se observa cómo también aumenta la tasa de éxito.

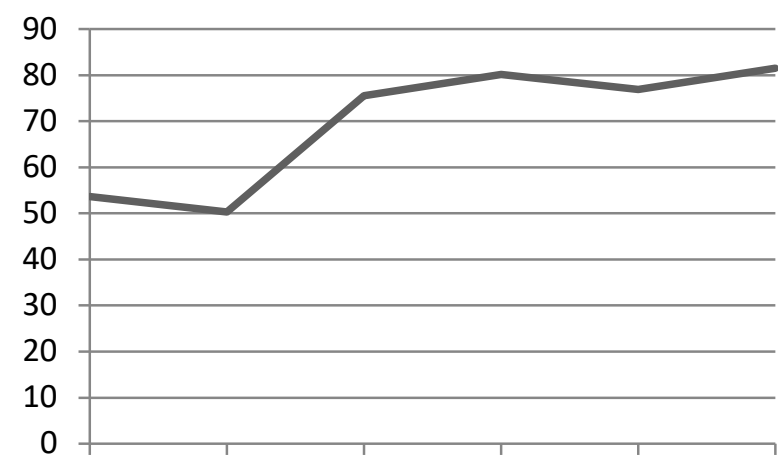

2011/12 2012/13 2013/14 2014/15 2015/16 2016/17

Figura 2. Evolución tasa de éxito de Expresión Gráfica en tanto por ciento. Fuente elaboración propia a partir de datos de los informes de la comisión de coordinación académica de curso de los años 2013 a 2017.

En cuanto a la tasa de absentismo en la Figura 3 se puede observar que muestra valores poco estables con grandes variaciones. Consideramos que los valores no son representativos pues no se encuentra la existencia de una relación evidente entre la tasa de absentismo y la implantación del proyecto monitor.

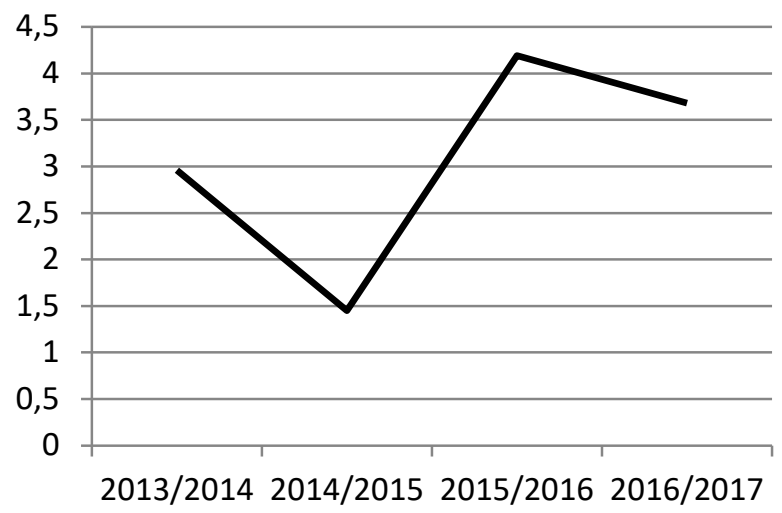

Figura 3. Evolución tasa de absentismo de Expresión Gráfica en tanto por ciento. Fuente elaboración propia a partir de datos de los informes de la comisión de coordinación académica de curso de los años 2013 a 2017.

En estudios realizados previamente sobre esta problemática (Álvarez, Cabrera, González y Bethencourt, 2006) Se han encontrado como variables causantes del abandono: la baja autoeficiencia, la baja motivación, las bajas expectativas sobre los resultados académicos, dificultades en la transición de etapa educativa, etc. (23 Pérez, Pedro R Álvarez 2011)

Respecto a la opinión de los alumnos sobre las mejores opciones para mitigar el abandono y el absentismo, una pauta importante es que hay controversia en muchos factores [...]. La única medida que parece haber despertado cierto consenso es la formación previa. Los alumnos encuestados apoyan decididamente que con este recurso se podría combatir el abandono. (Cernuda del Río, Agustín 2013)

Es por ello que consideramos que este valor podría mejorar si además de proporcionar una metodología por la cual se suplan las carencias en los conocimientos de partida de los alumnos se consiguiese trabajar en la motivación y el resto de 
factores que llevan a los alumnos al abandono de una asignatura.

Si estudiamos las notas de acceso de los alumnos del grado en Ingeniería de la Energía podemos observar en la Tabla 3 cómo se produce una bajada generalizada en la nota de acceso.

Tabla 3

Evolución temporal de las notas de acceso de los alumnos matriculados en el Grado de Ingeniería de la energía. Fuente elaboración propia a partir de datos facilitados por la UPM.

\begin{tabular}{cccccc}
\hline \multicolumn{6}{c}{ GRADO EN INGENIERIA DE LA ENERGIA } \\
Alumnos Matriculados \\
\hline $5-5,9$ & $6-7,9$ & $8-9,9$ & $10-11,9$ & $12-14$ \\
\hline $2011-2012$ & $0.8 \%$ & $0.0 \%$ & $5.6 \%$ & $89.5 \%$ & $4.0 \%$ \\
$2012-2013$ & $0.0 \%$ & $2.8 \%$ & $2.8 \%$ & $80.1 \%$ & $14.2 \%$ \\
$2013-2014$ & $0.0 \%$ & $1.6 \%$ & $3.1 \%$ & $81.9 \%$ & $13.4 \%$ \\
$2014-2015$ & $0.0 \%$ & $0.0 \%$ & $3.8 \%$ & $81.1 \%$ & $15.2 \%$ \\
$2015-2016$ & $0.6 \%$ & $0.6 \%$ & $37.6 \%$ & $55.8 \%$ & $5.5 \%$ \\
$2016-2017$ & $0.6 \%$ & $1.3 \%$ & $50.6 \%$ & $43.1 \%$ & $4.4 \%$ \\
\hline
\end{tabular}

También se observa en la Tabla 4 como esto sucede en el Grado de Ingeniería en Tecnología Minera.

\section{Tabla 4}

Evolución temporal de las notas de acceso de los alumnos matriculados en el Grado de Ingeniería en Tecnología Minera. Fuente elaboración propia a partir de datos facilitados por la UPM.

\begin{tabular}{cccccc}
\hline \multicolumn{6}{c}{ GRADO EN INGENIERIA DE MINAS } \\
Alumnos Matriculados \\
\hline $2011-2012$ & $0.0 \%$ & $50.7 \%$ & $42.7 \%$ & $6.0 \%$ & $0.7 \%$ \\
$2012-2013$ & $0.7 \%$ & $55.8 \%$ & $32.6 \%$ & $10.1 \%$ & $0.7 \%$ \\
$2013-2014$ & $4.6 \%$ & $50.5 \%$ & $38.8 \%$ & $6.1 \%$ & $0.0 \%$ \\
$2014-2015$ & $11.2 \%$ & $52.0 \%$ & $32.1 \%$ & $4.1 \%$ & $0.5 \%$ \\
$2015-2016$ & $14.5 \%$ & $52.7 \%$ & $24.8 \%$ & $7.9 \%$ & $0.0 \%$ \\
$2016-2017$ & $24.5 \%$ & $51.7 \%$ & $19.9 \%$ & $2.6 \%$ & $1.3 \%$ \\
\hline
\end{tabular}

Por último, se realizó un estudio comparativo en la evolución de la tasa de rendimiento de los dos grados (GIE y GITM) con los datos actuales disponibles, intentando reducir la influencia de factores que pueden intervenir en dicha tasa y pudiendo así estudiar el comportamiento de dichas asignaturas frente al Proyecto Monitor.

Con el objetivo de quitar el efecto de la "calidad” de los alumnos en cada año, se restó a cada tasa anual el promedio de dicha tasa en todas las asignaturas de ese año. Los valores obtenidos nos sirven para poder comparar asignaturas en un mismo año, valores positivos significarían que la asignatura se comporta mejor que las otras y viceversa. Ahora bien, nuestra intención es comparar la evolución de cada asignatura en los diferentes cursos en una escala normalizada para todas las asignaturas. Para ello, se restó a esos valores su valor promedio de los diferentes cursos para cada asignatura. El índice obtenido que llamaremos "tasa de rendimiento normalizada" nos sirve para valorar la evolución en el comportamiento de las asignaturas, habiendo sustraído el efecto de la "calidad" de los alumnos y pudiendo comparar entre asignaturas.

\section{Tabla 5}

Evolución de la tasa de rendimiento normalizada en el Grado de Ingeniería en Tecnología Minera. Fuente elaboración propia a partir de datos facilitados por la UPM.

\begin{tabular}{lccccc}
\hline \multicolumn{5}{c}{ GITM } \\
\hline & 2012 & $2013-$ & $2014-$ & $2015-$ & $2016-$ \\
& 2013 & 2014 & 2015 & 2016 & 2017 \\
\hline Algebra & -2.34 & -5.82 & -4.38 & 5.91 & 6.63 \\
Calculo I & 10.78 & 3.43 & -6.82 & -3.63 & -3.76 \\
Expresión & -20.41 & 9.13 & 23.72 & 6.16 & $--*$ \\
grafica & 5.17 & 8.94 & 2.08 & -10.45 & -5.74 \\
Física I & -8.23 & 8.95 & 1.43 & 5.33 & -7.47 \\
Informática & 6.02 & -3.24 & -3.75 & -0.45 & 1.42 \\
Química I & 34.59 & -15.86 & -12.78 & -5.95 & $--*$ \\
Calculo II & -26.33 & -4.59 & 19.57 & 11.34 & $--*$ \\
Estadística & -2.31 & -0.33 & -1.92 & 4.55 & $--*$ \\
Física II & -2.95 & 6.27 & -10.27 & -5.94 & $--*$ \\
Química II & 9.95
\end{tabular}

Nota: --* los valores del curso 2016/2017 de las asignaturas correspondientes al segundo semestre no se encuentran disponibles.

Las tablas 5 y 6 muestran la evolución de la tasa de rendimiento normalizada en el caso del Grado de Ingeniería de la energía y Grado de Ingeniería en Tecnología Minera. Lo interesante en dichas tablas es ver tendencias de crecimiento en cada asignatura.

\section{Tabla 6}

Evolución de la tasa de rendimiento normalizada en el Grado de Ingeniería de la energía. Fuente elaboración propia a partir de datos facilitados por la UPM.

\begin{tabular}{lccccc}
\hline \multicolumn{5}{c}{ GIE } & \\
\hline & 2012 & 2013 & 2014 & 2015 & 2016 \\
& 2013 & 2014 & 2015 & 2016 & 2017 \\
\hline Algebra & -2.95 & -8.39 & -3.28 & 10.07 & 4.56 \\
Calculo I & 12.73 & 4.64 & -5.93 & 1.81 & -13.25 \\
Expresión & -20.00 & -3.33 & 3.97 & 10.38 & 8.99 \\
grafica & -1.05 & 8.27 & 5.50 & 0.97 & -13.71 \\
Física I & 2.97 & -2.91 & 4.69 & -0.52 & -4.25 \\
Química I & 15.03 & -4.07 & -14.30 & 3.33 & $--*$ \\
Calculo II & -18.40 & 9.12 & 15.39 & -6.12 & $--*$ \\
Estadística & 13.56 & 0.67 & -3.77 & -10.45 & $--*$ \\
Física II & -7.75 & 0.07 & 3.63 & 4.05 & $--*$ \\
Informática & 10.27 & 0.34 & -1.50 & -9.10 & $--*$ \\
Química II & 10.27
\end{tabular}

Nota: --* los valores del curso 2016/2017 de las asignaturas correspondientes al segundo semestre no se encuentran disponibles.

Valores mayores que el curso anterior significan que la asignatura se ha comportado mejor (independientemente del 
efecto de la “calidad” de los alumnos). Si centramos la atención en las 2 asignaturas que se implantó el Proyecto Monitor en 3 cursos consecutivos (Expresión Gráfica y Química II) podemos observar cómo Expresión Gráfica muestra un crecimiento claro tanto en el Grado de Ingeniería de la energía como en Grado de Ingeniería en Tecnología Minera, exceptuando el curso 2015-2016 de GITM. De hecho, es la asignatura con mayor crecimiento de los grados de la ETSIME. Por otro lado, Química II no refleja ese buen comportamiento y se observa un decrecimiento del índice. Estos resultados hacen pensar que, aunque el Proyecto Monitor se establece como una medida positiva en el aprendizaje de los alumnos, es clave su forma de implantación, siendo el trabajo en el aula con la colaboración profesorado-alumnos fundamental para que el sistema de evolución continua dé los resultados esperados. Se espera que los cursos venideros, implantando este último sistema, confirmen los datos tan prometedores observados en la asignatura de Expresión Gráfica.

\section{CONCLUSIONES}

Como se observa en los resultados obtenidos la nota de acceso a los grados de estudio ha disminuido considerablemente a los largo de los años, esto se traduce en una menor preparación en cuanto a conocimientos previos por parte de los alumnos de nuevo ingreso lo que incrementa la dificultad de superar los primeros cursos satisfactoriamente.

Si relacionamos estos datos con la tasa de rendimiento podemos observar una correlación entre esta bajada generalizada de nivel y los valores de la tasa de rendimiento obtenida. Por ejemplo, en la asignatura de Cálculo I la tasa de rendimiento desciende hasta la mitad, sin embargo en la asignatura de Expresión Gráfica no solo no se produce este descenso sino que se produce un incremento de esta tasa casi en un 30\%. Esta asignatura es una de las que se enmarcan en el Programa del Proyecto monitor siguiendo el formato en el que el refuerzo de conocimiento se realiza en el aula.

En la asignatura de Química I la cual también se enmarca en este proyecto se puede observar que aunque se ha producido un descenso en esta tasa este descenso se ha conseguido paliar en alguno de los cursos. La diferencia entre estas dos asignaturas reside en el formato que llevan a cabo del proyecto siendo la asignatura de Química I una en las que se ha implantado el segundo formato de refuerzo de conocimiento en horario no lectivo. Esto supone cierto grado de compromiso por parte de los alumnos que hasta el momento no se ha conseguido. Esta falta de compromiso impide que el proyecto se lleve a cabo de una forma satisfactoria y por lo tanto los resultados obtenidos no han supuesto un cambio significativo como es el caso de Expresión Gráfica.

En el curso 2016/2017 se ha observado una mejora en el nivel de los alumnos de Química II que será estudiada en profundidad estando en este momento a la espera de los resultados. Esta mejora podría suponer que el refuerzo debería concentrarse en la asignatura del primer semestre en la cual se podrían paliar las deficiencias que los alumnos presentan.

Tras correlacionar los datos y observar las relaciones establecidas entre los diferentes indicadores, podemos concluir que los resultados obtenidos en la asignatura de Expresión Gráfica son satisfactorios, pues podemos observar que a pesar de encontrarnos en un momento en el que la nota de acceso de los alumnos y por lo tanto su nivel de entrada es cada vez más bajo, se está consiguiendo elevar tanto la tasa de éxito como la de rendimiento.

Del estudio realizado también podemos deducir que existe una notable diferencia entre los distintos formatos que han sido implantados en las asignaturas. El formato en el que el refuerzo de conocimiento se realiza en las aulas obtiene mejores resultados que el formato desarrollado fuera del aula con tutorización del profesorado.

Los resultados encontrados en este estudio previo nos hace ser muy optimista con las posibilidades del proyecto. En años posteriores estudiaremos a que son debidas las diferencias en la metodología utilizada y cuál es el factor de impacto que tiene la figura del profesor coordinador de la asignatura o la importancia del rollo del alumno tutor.

\section{REFERENCIAS}

Cabrera, E. M., García, L. A. G., \& Hernández, P. H. (1999). Determinantes de éxito y fracaso en la trayectoria del estudiante universitario. Servicio de Publicaciones, Universidad

Cernuda del Río, A. (2013). Un estudio sobre el absentismo y el abandono en asignaturas de programación. Revisión, 6(1).

García, A. M. D., Bravo, R. B., Albero, J. G., Cuello, R. O., \& Sancho, L. S. (2005). Competencias y diseño de la evaluación continua y final en el Espacio Europeo de Educación Superior. Programa de estudios y análisis.

Pérez, P. R. Á., \& AGUILAR, Y. D. L. (2011). El absentismo en la enseñanza universitaria: un obstáculo para la participación y el trabajo autónomo del alumnado. Bordón, 63(3), 43-56.

Reyes Ruiz-Gallardo, J., \& Castaño, S. (2008). La universidad española ante el reto del EEES.

Sánchez García, M., Manzano Soto, N., Rísquez, A., \& Suárez Ortega, M. (2011). Evaluación de un modelo de orientación tutorial y mentoría en la Educación Superior a distancia.

Universidad Politécnica de Madrid (2014-2017). Informe de la comisión de coordinación académica de curso. 